\title{
Saving the Species
}

\author{
Chris Bagnall
}

(C) Elsevier Science Publishing Co., Inc., 1986

For eighteen volumes, the cover of this journal has carried an illustration of a pair of tetrakaidecahedra, crystal forms found in an ideal polycrystalline material, completely and uniformly filling three-dimensional space. It is difficult to believe, but as-drawn, these polyhedra are sadly lacking in proper dimensional representation, and packing them together, without intercrystalline voids, would be an impossible task!

Your reaction to this may well be... "so what? - I understand what the figures represent; they illustrate a scientific fact and I know these solid forms perfectly fill the space they occupy when packed together..." You may even have found one down a microscope, as Mr. Pawel was fortunate enough to do some time ago [1]; although this was imperfect too, I am sure your faith in its perfect shape was not shaken.

It is certainly true that the cuboctahedra have served their purpose well for a long time- - both on the cover of this journal and for the International Metallographic Society. But now we know that their representation is incorrect there is only one course of action to take-and that is to

Reprinted from Metallography, Vol. 19, Chris Bagnall, Saving the Species, pages 1-4, Copyright 1986, with permission from Elsevier.

Editor's Note The Metallography, Microstructure, and Analysis Advisory Editor Chris Bagnall wrote this article as an Editorial for Metallography, the predecessor of Materials Characterization, which was at the time the official journal of the International Metallographic Society. It is appropriate that we include this article in the inaugural issue of MMA, as it gives the background of our society's logo.

C. Bagnall ( $\square)$

MCS Associates, Inc., 637 Donohoe Road,

Latrobe, PA 15650, USA

e-mail: chris.mcs@productevaluationsystems.com make our mark a perfect one. The logo should do more than convey an idea; it should be a definitive statement in itself. The analogy between a polycrystalline metal with perfect crystallization and the study of metallurgy is too close; reaching for complete understanding between a world of researchers in one discipline, we are endeavoring to make sure that no gaps or voids remain as knowledge is gained and exchanged. Our science may never reach this perfect state, but at the very least we should have a clear picture of our goal in front of us.

Several months ago, the immediate past President of IMS, Dr. J. E. Bennett, received a letter from Dr. Branko Grünbaum, a mathematician at the University of Washington. Dr. Grünbaum wrote:

On a recent visit to our Engineering Library I happened to notice a recent issue of "Metallography." What attracted my attention was the drawing of two cuboctahedra on its cover which, as the explanation inside makes clear, is the logo of the International Metallographic Society; the two cuboctahedra are supposed to be Archimedean, with regular polygons as faces. Unfortunately, the drawing on the cover as well as the one inside are drawn terribly poorly...

He went on to explain that on the projection, it should be possible to draw families of lines, which should be parallel, since they represent images of mutually parallel lines on the figure. As you can see, for the two families shown, this is sadly not the case:

Dr. Grünbaum continued:

This letter is part of my effort to convince colleagues - mathematicians and others-that we should pay more than lip service to statements about the importance of geometry and spatial intuition. 


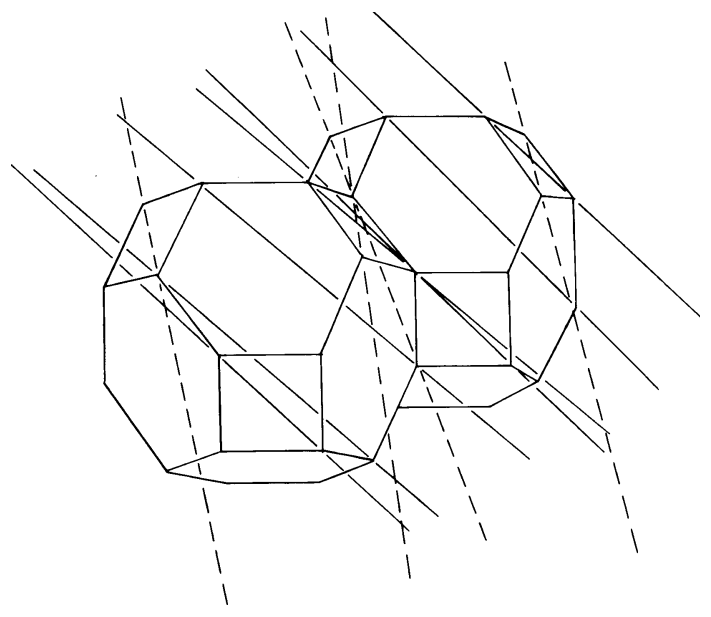

Fig. 1 The old logo-unparalleled distortion.

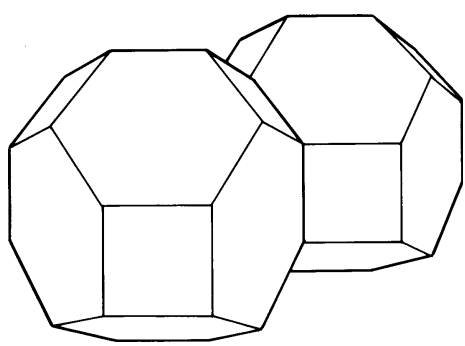

Fig. 2 The square face cannot be square-unless you are directly above it.

Dr. Grünbaum has been collecting incorrectly drawn icosahedra and other forms for some time, but his crusade to revive Euclid and bring Geometry back to our lives really picked up momentum when he discovered that the logo for his own society, the Mathematical Association of America, had been replaced with a defective version"which has been gracing Mathematics Magazine since January 1972!" A recent article of his in that journal [2] brings this and other travesties to light. It is a delightful article, and an eye-opener for all those of us who take pride in, and depend on, our powers of observation of fine detail for our livelihood. Dr. Grünbaum's conclusion should be taken to heart by us all:

Time is running out. Unless there is a strong general outcry against the continued visual abuse of the few remaining specimens, the endangered species, Homo Geometricus will surely vanish. That will be the end of a long era and YOU will be the poorer for it...

Understanding what is wrong with our logo is one thing; putting it right turned out to be quite another matter. There is a great deal to learn about what can and cannot be done with orthogonal, isometric, trimetric, multiple or singlepoint projection drawings as far as creating an accurate projection of a geometric form on a piece of paper in concerned. During a period of trial and error I was

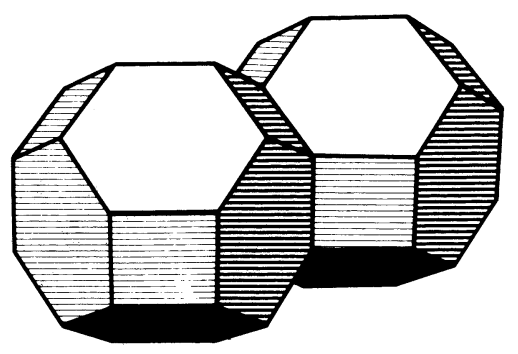

Fig. 3 Computer-aided perfection, and a pleasing perspective.

fortunate to have the assistance of a talented graphic artist, Duane Eisele, who suffered with me through a learning curve. We learned that an aesthetically pleasing figure was not necessarily right geometrically. Aesthetics do play an important role; the drawing can be correct but will appear distorted if the point of projection is too near or too far, too low or too much to one side. We came close, with a singlepoint projection, which is a commonly used format to display block shapes or scenes and is based on a copyrighted grid that has an undistorted square grid plane perpendicular to the viewer. Grid planes in the other dimensions expand away from this plane, but all four of these grids are different, so as to give the desired graphical effect. But, as our mentor, Dr. Grünbaum, was quick to point out you cannot look at the nearest square face of our tetrakaidecahedron, see it as truly square, and at the same time see more of the hexagon that has a common side with the top edge of the square than the hexagon at the bottom.

Dr. Grünbaum offered that at this degree of complication, drawings are difficult to execute accurately and it is safer to turn to a computer. So, without relying on any geometric or drafting construction, he instructed his computer to truncate the vertices of two regular octahedra (by far the simplest way of creating a tetrakaidecahedron) and then after the coordinates of a projection of similar aspect to our present logo had been determined the figure was drawn. And here it is.

The viewer is directly in front of, and lower than, the nearest square face. This is therefore rectangular with foreshortening on the sides. Note the "square" face on the adjacent tetrakaidecahedron is not even rectangular, because of the angular perspective.

As a conclusion to this editorial, now that you are beginning to feel comfortable that we finally have our logo drawn correctly and in so doing have contributed to the preservation of the endangered species Homo Geometricus, there is one more article you should read-by Dr. Grünbaum and co-authored by G.C. Shephard from the University of East Anglia, UK [3]. This paper discusses space filling with identical symmetrical solids. Three examples are wellknown; the cube, the rhombic dodecahedron and (our own) truncated octahedron. A fourth, a tetrahedron with bevelled 
vertices also exists. The question as to whether these four are the only forms depends on more finely-drawn lines of definition regarding "symmetrical" and "solid." One can only conclude after reading this article, and after living with an incorrect logo for eighteen years, that it is all too easy to accept an erroneous view or an incomplete picture, even when the facts are staring you in the face. We should be on our guard to not only look at all things in the right perspective, but to view them from many angles to make sure our understanding is complete.

\section{References}

1. C. Bagnall, It's the Real Thing! Editorial. Metallography 14, 1-2 (1981)

2. B. Grünbaum, Geometry Strikes Again. Mathematics Magazine 58, 12-17 (1985)

3. Branko. Grünbaum, G.C. Shephard, Space Filling with Identical Symmetrical Solids. The Mathematical Gazette 69, 117-120 (1985) 